Bull. Soc. math. France

133 (3), 2005, p. 395-417

\title{
VITESSE DANS LE THÉORÈME LIMITE CENTRAL POUR CERTAINS SYSTĖMES DYNAMIQUES QUASI-HYPERBOLIQUES
}

\author{
par Stéphane Le Borgne \& Françoise Pène
}

\begin{abstract}
RÉsumé. - Nous présentons une méthode permettant d'établir le théorème limite central avec vitesse en $n^{-1 / 2}$ pour certains systèmes dynamiques. Elle est basée sur une propriété de décorrélation forte qui semble assez naturelle dans le cadre des systèmes quasi-hyperboliques. Nous prouvons que cette propriété est satisfaite par les exemples des flots diagonaux sur un quotient compact de $\mathrm{SL}(d, \mathbb{R})$ et les «transformations » non uniformément hyperboliques du tore $\mathbb{T}^{3}$ étudiées par Shub et Wilkinson.
\end{abstract}

ABSTRACT (Rate of convergence in the central limit theorem). - We present a method which enables to establish the central limit theorem with rate of convergence in $n^{-1 / 2}$ for certain dynamical systems. It is based on a strong decorrelation property that seems to be quite natural for quasi-hyperbolic systems. We prove that this property is satisfied by the diagonal flows on a compact quotient of $\mathrm{SL}(d, \mathbb{R})$ and the non uniformly hyperbolic transformations of the torus $\mathbb{T}^{3}$ studied by Shub and Wilkinson.

Texte reçu le 12 juin 2003, accepté le 7 novembre 2003

Stéphane Le Borgne, Université de Rennes, Campus de Beaulieu, 35042 Rennes Cedex (France) • E-mail : sleborgn@univ-rennes1.fr

Françorse Pène, Université de Bretagne Occidentale, UFR Sciences et Techniques, Département de Mathématiques, 29285 Brest Cedex (France)

E-mail : francoise.pene@univ-brest.fr

Classification mathématique par sujets (2000). - 37D30, 22E46, 22F30, $60 \mathrm{~F} 05$.

Mots clefs. - Hyperbolicité partielle, quasi-hyperbolicité, théorème limite central. 


\section{Introduction}

Ce travail est une contribution à l'étude des propriétés stochastiques des systèmes dynamiques déterministes. Le problème général est le suivant. On se donne :

- un système dynamique probabilisé $(\Omega, \mathcal{F}, \nu, T)$, c'est-à-dire un espace $\Omega$ muni d'une tribu $\mathcal{F}$, une mesure de probabilité $\nu$ définie sur $\mathcal{F}$ et une transformation mesurable $T: \Omega \rightarrow \Omega$ préservant la mesure $\nu$,

- une fonction $f$ définie sur $\Omega$ à valeurs réelles,

et on cherche à décrire les propriétés du processus stationnaire $\left(f \circ T^{n}\right)$. On sait maintenant depuis longtemps que certaines propriétés d'hyperbolicité du système permettent de mettre à profit les méthodes probabilistes pour étudier le comportement asymptotique du processus $\left(f \circ T^{n}\right)(\mathrm{cf} .[12],[27],[31],[9]$, [4]). La décroissance des corrélations (cf. [7], [31], [3]), le théorème limite central (TLC) (cf. [30], [27], [31]), les principes d'invariance de Donsker et Strassen (cf. [4], [6], [11], [19], [23]) ont fait l'objet de nombreux articles.

Nous nous intéressons ici à la question de la vitesse de convergence dans le TLC. Établir le TLC pour une fonction $f$, c'est montrer l'existence d'une constante $\sigma>0$ telle que la suite $\left(1 /(\sigma \sqrt{n}) \sum_{k=0}^{n-1} f \circ T^{k}\right)_{n \geq 1}$ converge en loi vers une variable aléatoire gaussienne centrée réduite $N$. La vitesse de convergence dans le TLC s'obtient en majorant en fonction de $n$ la quantité

$$
\sup _{x \in \mathbb{R}}\left|\nu\left(\frac{1}{\sigma \sqrt{n}} \sum_{k=1}^{n} f \circ T^{k} \leq x\right)-\mathbf{P}(N \leq x)\right|
$$

Lorsque le système $(\Omega, \mathcal{F}, \nu, T)$ est un système d'Anosov, on peut établir (grâce à la technique basée sur la perturbation d'opérateur développée dans [12] ou [14]) une vitesse en $n^{-\frac{1}{2}}$, donc aussi bonne que celle obtenue par Esseen [10] pour les suites de variables aléatoires indépendantes.

Dans le cas des suites de différences de martingales, les vitesses que l'on peut obtenir dépendent d'hypothèses de moments (cf. [2], [13]). Il semble difficile d'appliquer ces méthodes dans les cas qui nous occupent ici. D'abord l'action de la transformation sur les fonctions régulières ne fait pas apparaître directement une suite de différences de martingale : il faut compter avec un terme pertubateur de type cobord. Ensuite les résultats de [2] et [13] comportent des conditions de contrôle non-stationnaires qui sont évidemment violées dans les cas qui nous intéressent. Par exemple, la vitesse en $n^{-\frac{1}{2}}$ prouvée dans [2] l'est sous une hypothèse portant sur les cubes des variables qui n'est généralement pas satisfaite dans notre cadre.

En toute généralité, la décroissance exponentielle des corrélations n'entraîne pas le théorème limite central. Dans cet article nous nous proposons de montrer que :

TOME $133-2005-\mathrm{N}^{\mathrm{O}} 3$ 
- si on renforce convenablement cette propriété de décorrélation, alors on peut démontrer un résultat de vitesse de convergence en $n^{-\frac{1}{2}}$ dans le TLC ;

- ce renforcement peut être facile à obtenir (à partir de la propriété de décorrélation «classique » quand on en dispose).

Depuis les travaux de Esseen, différentes méthodes ont été employées pour obtenir une vitesse dans le TLC. Nous suivrons ici la méthode développée par Rio [28] (voir aussi [17], [15], [26]) .

Notations. - Tout au long de ce travail nous noterons $\mathbf{E}_{\nu}[$.$] l'espérance rela-$ tivement à la mesure $\nu$ :

$$
\mathbf{E}_{\nu}[f]:=\int_{\Omega} f \mathrm{~d} \nu
$$

Pour toutes fonctions $f, g$ dans $L^{2}(\Omega, \nu)$ à valeurs complexes, nous utiliserons les écritures suivantes :

$$
\langle f, g\rangle=\mathbf{E}_{\nu}[f g] \quad \text { et } \operatorname{Cov}_{\nu}(f, g)=\mathbf{E}_{\nu}[f g]-\mathbf{E}_{\nu}[f] \cdot \mathbf{E}_{\nu}[g] .
$$

Étant donnée une suite stationnaire de variables $\left(X_{k}\right)_{k \geq 0}$, pour tout entier naturel $n$, nous noterons

$$
S_{n}:=\sum_{k=1}^{n} X_{k}
$$

avec la convention $S_{0}=0$.

Le résultat suivant (prouvé dans [17]) résulte de la démonstration de Rio [28].

THÉORÈme 1 (voir [28]). — Soit $\left(X_{k}\right)_{k \geq 0}$ une suite stationnaire de variables aléatoires réelles bornées centrées définies sur un même espace probabilisé telle que, pour tous entiers naturels $a, b, c$ vérifiant $1 \leq a+b+c \leq 3$, la série suivante soit convergente :

(1) $\sum_{p \geq 1} p \sup _{\substack{k \geq 0 \\ q \geq p, r \geq p}}\left\|\mathbf{E}\left[X_{k+p}^{a} X_{k+q}^{b} X_{k+r}^{c} \mid X_{0}, \ldots, X_{k}\right]-\mathbf{E}\left[X_{k+p}^{a} X_{k+q}^{b} X_{k+r}^{c}\right]\right\|_{\infty}$.

Alors, la limite suivante existe :

$$
\sigma:=\lim _{n \rightarrow+\infty} \frac{1}{\sqrt{n}}\left(\mathbf{E}\left[S_{n}^{2}\right]\right)^{\frac{1}{2}}
$$

- Si $\sigma=0$, alors la suite $\left(S_{n}\right)_{n}$ est bornée dans $L^{2}$.

- Si $\sigma>0$, alors la suite de variables aléatoires $\left(S_{n} / \sqrt{n}\right)_{n \geq 1}$ converge en loi vers une variable aléatoire $N$ de loi normale centrée de variance $\sigma^{2}$ et il existe un nombre réel $R>0$ tel que, pour tout entier $n \geq 1$, on ait

$$
\sup _{x \in \mathbb{R}}\left|\nu\left(\frac{S_{n}}{\sqrt{n}} \leq x\right)-\mathbf{P}(N \leq x)\right| \leq \frac{R}{\sqrt{n}} .
$$

BULletin DE LA SOCIÉtÉ MATHÉMATIQUE DE FRANCE 
En étudiant en détail la démonstration de Rio, on s'aperçoit qu'il utilise en fait la propriété suivante (qui découle de l'hypothèse (1) et peut la remplacer dans l'énoncé de son théorème) :

Il existe une suite $\varphi_{p}$ telle que la série $\sum_{p \geq 1} p \cdot \varphi_{p}$ converge et telle que, pour toute fonction continue $F: \mathbb{R}^{4} \rightarrow \mathbb{R}$ et pour tous entiers $j \leq k \leq k+p \leq$ $k+q \leq k+r$, on ait

$$
\left|\operatorname{Cov}\left(F\left(S_{j-1}, X_{j}, X_{k}, X_{\ell}\right), X_{\ell+p}^{a} X_{\ell+q}^{b} X_{\ell+s}^{c}\right)\right| \leq\left\|F\left(S_{j-1}, X_{j}, X_{k}, X_{\ell}\right)\right\|_{L^{1}} \varphi_{p} .
$$

On peut encore affaiblir les hypothèses et montrer le résultat suivant.

THÉORÈme 2. - Soit $\left(X_{k}\right)_{k \geq 0}$ une suite stationnaire de variables aléatoires réelles bornées centrées définies sur un même espace probabilisé. Supposons qu'il existe trois nombres réels $C \geq 1, M \geq \max \left(1,\left\|X_{0}\right\|_{\infty}\right)$ et $r \geq 1$, et une suite de nombres réels $\left(\xi_{p, \ell}\right)_{p}$ vérifiant $\sum_{p>1} p \max _{\ell=0, \ldots, p / r} \xi_{p, \ell}<+\infty$ tels que, pour tous entiers naturels $a, b, c$ vérifiant $a+b+c \leq 3$, pour tous entiers $j, k, \ell, p, q, s$ vérifiant $1 \leq j \leq k \leq \ell \leq \ell+p \leq \ell+q \leq \ell+s$, pour toute fonction différentiable $F: \mathbb{R}^{4} \rightarrow \mathbb{R}$, nous ayons

$$
\begin{aligned}
& \left|\operatorname{Cov}\left(F\left(S_{j-1}, X_{j}, X_{k}, X_{\ell}\right), X_{\ell+p}{ }^{a} X_{\ell+q}{ }^{b} X_{\ell+s}{ }^{c}\right)\right| \\
& \leq C \cdot \xi_{p, s-p}\left(\left\|F\left(S_{j-1}, X_{j}, X_{k}, X_{\ell}\right)\right\|_{L^{1}}\right. \\
& \left.\quad+\left\|\sup _{\substack{|u|,|v| \leq M \\
|w|,|z| \leq M}}\left|D F\left(S_{j-1}+u, X_{j}+v, X_{k}+w, X_{\ell}+z\right)\right|_{\infty}\right\|_{L^{1}}\right),
\end{aligned}
$$

(en identifiant, pour tout $(u, v, w, z) \in \mathbb{R}^{4}, D F(u, v, w, z)$ à un vecteur de $\mathbb{R}^{4}$ et en notant $|.|_{\infty}$ la norme supérieure sur $\left.\mathbb{R}^{4}\right)$. Alors, on a les mêmes conclusions que dans le théorème 1.

C'est ce théorème que nous allons utiliser.

Donnons quelques exemples de suites de $\left(\xi_{p, \ell}\right)_{p, \ell}$ satisfaisant la condition du théorème :

- toute suite $\left(\xi_{p, \ell}\right)_{p, \ell}$ telle que $\xi_{p, \ell}=\xi_{p}$ avec $\sum_{p \geq 1} p \xi_{p}<+\infty$ convient (nous retrouvons ainsi le théorème de Rio);

- toute suite $\left(\xi_{p, \ell}\right)_{p, \ell}$ de la forme $\xi_{p, \ell}=\left(1+\ell^{\beta}\right) \delta^{p}$ (avec $\beta>0$ et $\left.\delta \in\right] 0,1[)$ convient;

- remarquons même que toute suite $\xi_{p, \ell}$ de la forme $\xi_{p, \ell}=\delta^{p} K^{\ell}$ (avec $\delta \in] 0,1[$ et $K \geq 1$ ) convient.

Nous ne donnons pas ici la démonstration du théorème 2 car elle est technique, longue et suit dans ses grandes lignes celle de Rio [28] (on trouvera une démonstration dans [20]). L'objet de cet article est de montrer dans deux situations concrètes comment ce théorème peut être appliqué pour obtenir une vitesse dans le TLC. 


\section{L'exemple des flots diagonaux}

1.1. Énoncé du résultat. - Soit un entier $d \geq 2$ et $\Gamma$ un réseau cocompact de $G:=\mathrm{SL}(d, \mathbb{R})$. Nous considérons l'espace quotient

$$
\Omega:=\mathrm{SL}(d, \mathbb{R}) / \Gamma=\{x \Gamma ; x \in G\} .
$$

Cet espace a une structure de variété différentiable. La mesure de Haar $\mu$ sur $G$ donne une mesure finie sur $\Omega=G / \Gamma$ invariante par translation à gauche que nous notons $\bar{\mu}$ et supposons normalisée $(\bar{\mu}(G / \Gamma)=1)$. Nous désignerons par $\bar{x}$ la classe à gauche modulo $\Gamma$ de l'élément $x$ de $G$. Soit $\left(T_{i}\right)_{i=1}^{d}$ une suite décroissante de $d$ nombres positifs non tous égaux à 1 dont le produit vaut 1 . Appelons $T$ la matrice

$$
T=\left(\begin{array}{ccccc}
T_{1} & & & & \\
& T_{2} & & 0 & \\
& & \ddots & & \\
& 0 & & T_{d-1} & \\
& & & & T_{d}
\end{array}\right) .
$$

Le groupe à un paramètre

$$
\left\{T^{t}=\left(\begin{array}{ccccc}
T_{1}^{t} & & & & \\
& T_{2}^{t} & & 0 & \\
& & \ddots & & \\
& 0 & & T_{d-1}^{t} & \\
& & & & T_{d}^{t}
\end{array}\right) ; t \in \mathbb{R}\right\}
$$

définit sur $\Omega$ un flot, noté encore $T^{t}$, préservant la mesure $\bar{\mu}$ :

$$
T^{t}: G / \Gamma \longrightarrow G / \Gamma, \quad \bar{x} \longmapsto T^{t} \bar{x} .
$$

Fixons une distance riemannienne $d_{0}$ sur $G$ invariante par translation à droite et définissons une distance $d$ sur $\Omega$ en posant

$$
d(\bar{x}, \bar{y})=\inf _{\gamma \in \Gamma} d_{0}(x, y \gamma) .
$$

ThÉORÈme 3. - Soit $F: \Omega \rightarrow \mathbb{R}$ une fonction höldérienne, $\bar{\mu}$-centrée. Alors, la limite suivante existe :

$$
\sigma:=\lim _{t \rightarrow+\infty}\left(\mathbf{E}_{\bar{\mu}}\left[\left(\frac{1}{\sqrt{t}} \int_{0}^{t} F \circ T^{s} \mathrm{~d} s\right)^{2}\right]\right)^{\frac{1}{2}} .
$$

- Si $\sigma=0$, alors la suite de v.a. $\left(\int_{0}^{t} F \circ T^{s} \mathrm{~d} s\right)_{t>0}$ est bornée dans $L^{2}$.

- Si $\sigma>0$, alors la suite de variables aléatoires $\left(1 / \sqrt{t} \int_{0}^{t} F \circ T^{s} \mathrm{~d} s\right)_{t>0}$ converge en loi relativement à la mesure de probabilité $\bar{\mu}$ (lorsque $t$ tend vers $+\infty$ ) vers une variable aléatoire $N$ de loi normale centrée de variance $\sigma^{2}$ et il existe un nombre réel $R>0$ tel que, pour tout nombre réel $t \geq 1$, on ait

$$
\sup _{x \in \mathbb{R}}\left|\bar{\mu}\left(\frac{1}{\sqrt{t}} \int_{0}^{t} F \circ T^{s} \mathrm{~d} s \leq x\right)-\mathbf{P}(N \leq x)\right| \leq \frac{R}{\sqrt{t}} .
$$

BULletin DE LA SOCiÉtÉ MATHÉMATIQUE DE FRANCE 
Signalons que la même démonstration sera valable dans les situations où l'on dispose des mêmes outils, c'est-à-dire :

- la structure produit de la mesure invariante $\nu$;

- la régularité du jacobien de l'application d'holonomie sur les feuilles instables le long des feuilles stables-neutres ( $c f$. les définitions du $§ 1$ );

- une forme d'invariance par $T$ des mesures conditionnelles de $\nu$ le long des feuilles instables.

En particulier, on établit de la même manière un théorème analogue dans chacun des cas suivants :

- automorphismes ergodiques des tores;

- temps 1 du flot géodésique sur une surface riemannienne de courbure strictement négative;

- certaines transformations affines partiellement hyperboliques de nilvariétés traitées dans [5].

Pour l'exemple des flots diagonaux nous allons voir que l'hypothèse de décorrélation forte du théorème 2 est satisfaite pour une suite $\left(\xi_{p, \ell}\right)_{p, \ell}$ de la forme $\xi_{p, \ell}=\delta^{p}$, c'est-à-dire indépendante de $\ell$. Il en est de même pour les autres exemples évoqués excepté les automorphismes ergodiques des tores : dans les cas où la restriction de la matrice définissant l'automorphisme du tore à sa feuille neutre comporte des blocs de Jordan non triviaux il est nécessaire de considérer une suite $\left(\xi_{p, \ell}\right)_{p, \ell}$ de la forme $\xi_{p, \ell}=\left(1+\ell^{\beta}\right) \delta^{p}$.

Remarque. - Posons $X_{k}:=\int_{k-1}^{k} F \circ T^{s} \mathrm{~d} s$ et $f(y)=\int_{-1}^{0} F \circ T^{s}(y) \mathrm{d} s$. Nous avons $X_{k}=f \circ T^{k}$. Il suffit de montrer que le théorème 2 s'applique pour la suite de variables aléatoires $\left(X_{k}\right)$. Si $F$ est höldérienne, $f$ l'est également.

\subsection{Quelques définitions}

Feuilletages. - Rappelons les propriétés hyperboliques du système dynamique $(\Omega, \bar{\mu}, T)$ avec $\Omega=\operatorname{SL}(d, \mathbb{R}) / \Gamma$ et $T=T^{1}$. Les relations

$$
\left(T x T^{-1}\right)_{i j}=\frac{T_{i} x_{i j}}{T_{j}}
$$

permettent d'identifier les variétés stables, instables et neutres du difféomorphisme $T$. Considérons la partition de $\{1, \ldots, d\}$ en les ensembles $J_{k}$ définis par

- pour tout $k$, pour tous $i, j$ appartenant à $J_{k}$, on a $T_{i}=T_{j}$;

- pour tous $k, n$ tels que $k<n$, pour tout $i$ appartenant à $J_{k}$, tout $j$ appartenant à $J_{n}$, on a $T_{i}>T_{j}$.

Désignons par $h_{J_{i} J_{j}}$ une matrice indexée par l'ensemble $J_{i} \times J_{j}$, et par $\operatorname{Id}_{J_{i}}$ la matrice identité indexée par $J_{i}$. Les variétés stables, instables et neutres de $T$ sont données par les orbites de groupes de matrices triangulaires ou diagonales par blocs.

TOME $133-2005-\mathrm{N}^{\mathrm{O}} 3$ 
La variété instable passant par $\bar{x}=x \Gamma$ est la variété immergée $H_{u} x \Gamma$ définie par le groupe $H_{u}$ des matrices de la forme

$$
h_{u}=\left(\begin{array}{ccccc}
\operatorname{Id}_{J_{1}} & h_{J_{1} J_{2}} & \ldots & h_{J_{1} J_{\ell-1}} & h_{J_{1} J_{\ell}} \\
0 & \operatorname{Id}_{J_{2}} & \ldots & h_{J_{2} J_{\ell-1}} & h_{J_{2} J_{\ell}} \\
\vdots & \vdots & \ddots & \vdots & \vdots \\
0 & 0 & \ldots & \operatorname{Id}_{J_{\ell-1}} & h_{J_{\ell-1} J_{\ell}} \\
0 & 0 & \ldots & 0 & \operatorname{Id}_{J_{\ell}}
\end{array}\right)
$$

De même la variété stable (resp. neutre) passant par $\bar{x}=x \Gamma$ est la variété immergée $H_{s} x \Gamma$ (resp. $H_{e} x \Gamma$ ) définie par le groupe $H_{s}\left(\right.$ resp. $\left.H_{e}\right)$ des matrices de la forme

$$
\begin{array}{r}
h_{s}=\left(\begin{array}{ccccc}
\operatorname{Id}_{J_{1}} & 0 & \ldots & 0 & 0 \\
h_{J_{2} J_{1}} & \operatorname{Id}_{J_{2}} & \ldots & 0 & 0 \\
\vdots & \vdots & \ddots & \vdots & \vdots \\
h_{J_{\ell-1} J_{1}} & h_{J_{\ell-1} J_{2}} & \ldots & \operatorname{Id}_{J_{\ell-1}} & 0 \\
h_{J_{\ell} J_{\ell}} & h_{J_{\ell} J_{2}} & \ldots & h_{J_{\ell} J_{\ell-1}} & \operatorname{Id}_{J_{\ell}}
\end{array}\right) \\
\\
\left(\operatorname{resp.} h_{e}=\left(\begin{array}{cccc}
h_{J_{1} J_{1}} & 0 & \ldots & 0 \\
0 & h_{J_{2} J_{2}} & \ldots & 0 \\
\vdots & \vdots & \ddots & \vdots \\
0 & 0 & \ldots & h_{J_{\ell} J_{\ell}}
\end{array}\right)\right)
\end{array}
$$

Rappelons que la distance $d$ sur $\Omega$ est définie à partir d'une distance riemannienne $d_{0}$ sur $G$ invariante par translation à droite en posant

$$
d(\bar{x}, \bar{y})=\inf _{\gamma \in \Gamma} d_{0}(x, y \gamma)
$$

Pour tous $x \in G, h_{u} \in H_{u}, h_{s} \in H_{s}, h_{e} \in H_{e}$, posons

$$
d^{u}\left(x \Gamma, h_{u} x \Gamma\right):=d_{0}\left(\mathrm{Id}, h_{u}\right), \quad d^{s, e}\left(x \Gamma, h_{s} h_{e} x \Gamma\right):=d_{0}\left(\mathrm{Id}, h_{s} h_{e}\right) .
$$

La quantité $d^{u}(\bar{x}, \bar{y})$ n'est définie que si $\bar{y}$ appartient à $H_{u} \bar{x}$. En restriction à $H_{u} \bar{x} \times H_{u} \bar{x}, d^{u}$ est une distance. On peut faire une remarque analogue pour $d^{s, e}$.

Grâce aux relations (2), on montre l'existence de constantes positives $C_{0}$ et $\delta>1$ telles que, pour tout $\bar{x} \in \Omega$ et tout entier $n \geq 0$, nous avons :

- une propriété de non-dilatation le long des feuilles stables-neutres: pour tout $\bar{y} \in H_{s} H_{e} \bar{x}$

$$
d^{s, e}\left(T^{n}(\bar{x}), T^{n}(\bar{y})\right) \leq C_{0} d^{s, e}(\bar{x}, \bar{y}) ;
$$

- une propriété de contraction le long des feuilles instables : pour tout $\bar{y} \in H_{u} \bar{x}$,

$$
d^{u}\left(T^{-n}(\bar{x}), T^{-n}(\bar{y})\right) \leq C_{0} \delta^{-n} d^{u}(\bar{x}, \bar{y})
$$

BULletin DE LA SOCiÉtÉ MATHÉmATiQUe DE FRANCE 
Régularité de l'holonomie le long des feuilles $H_{e} H_{s}$. — Désignons par $B(r)$ (resp. $\left.B_{u}(r), B_{e}(r), B_{s}(r)\right)$ les boules $\left\{h \in G ; d_{0}(\mathrm{Id}, h)<r\right\}\left(\operatorname{resp} . B(r) \cap H_{u}\right.$, $\left.B(r) \cap H_{e}, B(r) \cap H_{s}\right)$. Il existe un nombre réel $r_{1}>0$ tel que, pour tout $\bar{x}$, l'application

$$
\begin{aligned}
B_{u}\left(r_{1}\right) \times B_{e}\left(r_{1}\right) \times B_{s}\left(r_{1}\right) & \longrightarrow B_{u}\left(r_{1}\right) B_{e}\left(r_{1}\right) B_{s}\left(r_{1}\right) \bar{x}, \\
\left(h_{u}, h_{e}, h_{s}\right) & \longmapsto h_{u} h_{e} h_{s} \bar{x}
\end{aligned}
$$

soit un difféomorphisme.

Dans toute la suite, $r_{0}$ désigne un nombre réel fixé vérifiant $0<r_{0}<\frac{1}{3} r_{1}$,

$$
B_{s}\left(3 r_{0}\right) B_{e}\left(3 r_{0}\right) B_{u}\left(r_{0}\right) B_{e}\left(r_{0}\right) B_{s}\left(r_{0}\right) \subseteq B_{u}\left(r_{1}\right) B_{e}\left(r_{1}\right) B_{s}\left(r_{1}\right)
$$

et suffisamment petit pour que

$$
B_{u}(r) B_{e}(r) B_{s}(r) \bar{x}:\left(h_{u}, h_{e}, h_{s}\right) \longmapsto h_{u} h_{e} h_{s} \bar{x}
$$

soit un difféomorphisme local et ce qui suit soit vrai.

Considérons un point $\bar{x}$ quelconque de $G / \Gamma,\left(h_{e}, h_{s}, h_{e}^{\prime}, h_{s}^{\prime}\right)$ un élément de $B_{e}\left(r_{0}\right) \times B_{s}\left(r_{0}\right) \times B_{e}\left(r_{0}\right) \times B_{s}\left(r_{0}\right)$. Pour tout $h_{u} \in B_{u}\left(r_{0}\right)$, le morceau de variété stable-neutre $B_{s}\left(3 r_{0}\right) B_{e}\left(3 r_{0}\right) h_{u} h_{e} h_{s} \bar{x}$ rencontre le morceau de variété instable $B_{u}\left(2 r_{0}\right) h_{e}^{\prime} h_{s}^{\prime} \bar{x}$ en un point unique. Appelons $\mathcal{H}$ l'application qui à $h_{u} h_{e} h_{s} \bar{x}$ associe ce point d'intersection (pour simplifier l'écriture, nous ne retenons pas dans la notation que $\mathcal{H}$ dépend de $\left.\left(\bar{x}, h_{e}, h_{s}, h_{e}^{\prime}, h_{s}^{\prime}\right)\right)$. Par définition, $\mathcal{H}\left(h_{u} h_{e} h_{s} \bar{x}\right)$ est l'unique point qui puisse s'écrire de deux façons $\widetilde{h}_{e} \widetilde{h}_{s} h_{u} h_{e} h_{s} \bar{x}$ et $\widetilde{h}_{u} h_{e}^{\prime} h_{s}^{\prime} \bar{x}$ avec $\left(\widetilde{h}_{e}, \widetilde{h}_{s}, \widetilde{h}_{u}\right) \in B_{e}\left(3 r_{0}\right) \times B_{s}\left(3 r_{0}\right) \times B_{u}\left(2 r_{0}\right)$. Le triplet $\left(\widetilde{h}_{e}, \widetilde{h}_{s}, \widetilde{h}_{u}\right)$ est donc déterminé par

$\left(\widetilde{h}_{e}, \widetilde{h}_{s}, \widetilde{h}_{u}\right) \in B_{e}\left(3 r_{0}\right) \times B_{s}\left(3 r_{0}\right) \times B_{u}\left(2 r_{0}\right) \quad$ et $\quad \widetilde{h}_{u}^{-1} \widetilde{h}_{e} \widetilde{h}_{s}=h_{e}^{\prime} h_{s}^{\prime} h_{s}^{-1} h_{e}^{-1} h_{u}^{-1}$.

Ceci montre que l'application $\mathcal{H}$ est une fonction régulière de $\left(h_{e}, h_{s}, h_{e}^{\prime}, h_{s}^{\prime}\right)$. De plus la mesure sur $B_{u}\left(2 r_{0}\right) h_{e}^{\prime} h_{s}^{\prime} \bar{x}$ image par $\mathcal{H}$ de la mesure $\mu_{u}$ sur $B_{u}\left(r_{0}\right) h_{e} h_{s} \bar{x}$ est absolument continue par rapport à la mesure $\mu_{u}\left(\operatorname{sur} B_{u}\left(2 r_{0}\right) h_{e}^{\prime} h_{s}^{\prime} \bar{x}\right)$ et sa densité est elle aussi une fonction régulière de $\left(h_{e}, h_{s}, h_{e}^{\prime}, h_{s}^{\prime}\right)$. Il existe un nombre réel $C_{1}>0$ tel que, pour tout $\left(h_{u}, h_{e}, h_{s}, h_{e}^{\prime}, h_{s}^{\prime}\right)$ et tout $\bar{x}$, on ait

$$
d\left(\mathcal{H}\left(h_{u} h_{e} h_{s} \bar{x}\right), h_{u} h_{e} h_{s} \bar{x}\right) \leq C_{1} d\left(h_{e} h_{s} \bar{x}, h_{e}^{\prime} h_{s}^{\prime} \bar{x}\right)
$$

et

$$
\left|\frac{d \mathcal{H}\left(\mu_{u}\right)}{d \mu_{u}}\left(\mathcal{H}\left(h_{u} h_{e} h_{s} \bar{x}\right)\right)-1\right| \leq C_{1} d\left(h_{e} h_{s} \bar{x}, h_{e}^{\prime} h_{s}^{\prime} \bar{x}\right) .
$$

Identité approchée

DÉFInition 1.1. - Soient $\rho>1$ et $C_{2}>0$ deux nombres réels. Une $\left(\rho, C_{2}\right)$ identité approchée est une suite $\left(\chi_{n}\right)_{n \geq 0}$ de fonctions définies sur $G$, positives ou nulles, indéfiniment dérivables, d'intégrale 1 , telle que, pour tout entier naturel $n$, on ait

- le support de $\chi_{n}$ est inclus dans $B_{0}\left(\mathrm{Id}, \rho^{-n}\right)$;

TOME $133-2005-\mathrm{N}^{\mathrm{O}} 3$ 
- $\left\|\Delta \chi_{n}\right\|_{\infty} \leq C_{2} \rho^{C_{2} n}$;

- $\chi_{n}$ est lipschitzienne de constante de Lipschitz $\rho^{C_{2} n}$.

De telles suites existent. Soient $\left(\chi_{n}^{(\rho)}\right)_{n \geq 0}$ une $\left(\rho, C_{2}\right)$-identité approchée et $\widehat{\psi}$ une fonction localement intégrable sur $G$. Posons

$$
\chi_{n}^{(\rho)} * \widehat{\psi}(x)=\int_{G} \widehat{\psi}\left(g^{-1} x\right) \chi_{n}^{(\rho)}(g) \mathrm{d} \mu(g)=\int_{G} \widehat{\psi}(g) \chi_{n}^{(\rho)}\left(x g^{-1}\right) \mathrm{d} \mu(g) .
$$

Identifions l'algèbre de Lie de $G$ à l'ensemble des champs de vecteurs sur $G$ invariants à droite. Alors, pour tout entier naturel $n$, les fonctions $\chi_{n}^{(\rho)} * \widehat{\psi}$ sont indéfiniment différentiables et $\Omega\left(\chi_{n}^{(\rho)} * \widehat{\psi}\right)=\left(\Omega \chi_{n}^{(\rho)}\right) * \widehat{\psi}$ pour tout opérateur différentiel $\Omega$ appartenant à l'algèbre enveloppante universelle de $G$. Soit $\varphi$ une fonction $\bar{\mu}$-intégrable définie sur $G / \Gamma$. On lui associe une fonction $\widehat{\varphi}$ définie sur $G$ localement intégrable en posant $\widehat{\varphi}(x)=\varphi(\bar{x})$. On vérifie immédiatement (sur la première définition) que $\chi_{n}^{(\rho)} * \widehat{\varphi}$ est invariante par translation à droite par les éléments de $\Gamma$. Il lui correspond donc une fonction définie sur $G / \Gamma$ : nous la notons $\chi_{n}^{(\rho)} * \varphi$.

1.3. Mélange et décorrélation forte. - Grâce à la théorie des représentations des groupes de Lie, on peut établir le résultat suivant (voir [18], [19]) :

Proposition 1.2. - Il existe deux nombres réels $\zeta>1$ et $C_{3}>0$ et un opérateur différentiel $\Delta$ tels que, pour toutes fonctions $\varphi$ et $\psi$ indéfiniment dérivables définies sur $G / \Gamma$ d'intégrales nulles et pour tout entier $n$, nous avons

$$
\left|\left\langle\varphi, \psi \circ T^{n}\right\rangle\right| \leq C_{3}\|\Delta \varphi\|_{2} \cdot\|\Delta \psi\|_{2} \zeta^{-|n|} .
$$

Lorsqu'on applique une telle majoration pour tenter de vérifier l'hypothèse de décorrélation du théorème 2 , on obtient une inégalité sans intérêt car, même dans les cas où elles sont finies, les normes $\left\|\Delta F\left(S_{j-1}, X_{j}, X_{k}, X_{\ell}\right)\right\|_{2}$ et $\left\|\Delta X_{\ell+p}{ }^{a} X_{\ell+q}{ }^{b} X_{\ell+s}{ }^{c}\right\|_{2}$ croissent généralement exponentiellement vite avec les nombres $\ell-j$ et $s$.

La régularité des fonctions le long des feuilles instables s'améliore sous l'action de $T^{-1}$ et la régularité des fonctions le long des feuilles stables-neutres ne se détériore pas trop sous l'action de $T$. Cette remarque conduit à remplacer la norme apparaissant dans la formule (5) par deux normes différentes (l'une pour $\varphi$ mesurant la régularité des fonctions le long des feuilles instables, l'autre pour $\psi$ mesurant la régularité des fonctions le long des feuilles stables-neutres).

Pour tout nombre réel $\eta \in] 0 ; 1]$ et toute fonction $\varphi: G / \Gamma \rightarrow \mathbb{R}$, nous notons

- $C_{\varphi}^{(\eta)}$ le coefficient de Hölder d'ordre $\eta$ de $\varphi$ relativement à la distance $d$ :

$$
C_{\varphi}^{(\eta)}:=\sup _{\bar{x} \in \Omega} \sup _{\bar{y} \neq \bar{x}} \frac{|\varphi(\bar{x})-\varphi(\bar{y})|}{(d(\bar{x}, \bar{y}))^{\eta}}
$$


- $C_{\varphi}^{(\eta, s, e)}$ le coefficient de Hölder d'ordre $\eta$ de $\varphi$ dans la direction stableneutre :

$$
C_{\varphi}^{(\eta, s, e)}:=\sup _{\bar{x} \in \Omega} \sup _{\bar{y} \in H_{s} H_{e} \bar{x} \backslash\{\bar{x}\}} \frac{|\varphi(\bar{x})-\varphi(\bar{y})|}{\left(d^{s, e}(\bar{x}, \bar{y})\right)^{\eta}} ;
$$

- $C_{\varphi}^{(\eta, u)}$ le coefficient de Hölder d'ordre $\eta$ de $\varphi$ dans la direction instable :

$$
C_{\varphi}^{(\eta, u)}:=\sup _{\bar{x} \in \Omega} \sup _{\bar{y} \in H_{u} \bar{x} \backslash\{\bar{x}\}} \frac{|\varphi(\bar{x})-\varphi(\bar{y})|}{\left(d^{u}(\bar{x}, \bar{y})\right)^{\eta}} ;
$$

- $\|\cdot\|_{(\eta, s, e)}$ la « norme » de Hölder

$$
\|\cdot\|_{(\eta, s, e)}:=\|\cdot\|_{\infty}+C^{(\eta, s, e)}
$$

- $D_{\varphi}^{(\eta, u, L)}$ une notion de coefficient de Hölder local dans la direction instable :

$$
D_{\varphi}^{(\eta, u, L)}(\bar{x}):=\sup _{\substack{\bar{y} \in H_{u} \bar{x} \\ d^{u}(\bar{x}, \bar{y}) \leq L}} \frac{|\varphi(\bar{x})-\varphi(\bar{y})|}{\left(d^{u}(\bar{x}, \bar{y})\right)^{\eta}} .
$$

LEMME 1.3.1. - S'il existe des nombres réels $C_{4}>1, L_{0}$ et $\left.\alpha_{0} \in\right] 0,1[$ tels que, pour toutes fonctions $\varphi$ et $\psi$, et tout entier $n \geq 1$, nous ayons

$$
\left|\operatorname{Cov}_{\nu}\left(\varphi, \psi \circ T^{n}\right)\right| \leq C_{4}\left(\|\varphi\|_{L^{1}}\|\psi\|_{(\eta, s, e)}+\|\psi\|_{\infty}\left\|D_{\varphi}^{\left(\eta, u, L_{0}\right)}\right\|_{L^{1}}\right) \alpha_{0}^{n}
$$

alors, si $f$ est une fonction höldérienne, bornée et centrée, l'hypothèse du théorème 2 est valable pour la suite de variables aléatoires $\left(X_{k}=f \circ T^{k}\right)_{k \geq 0}$.

La condition (6) du lemme est a priori plus forte que le mélange exponentiel. À notre connaissance, Bressaud et Liverani [3] sont les seuls à avoir obtenu une majoration de ce type (pour les difféomorphismes d'Anosov, voir aussi [1]). Il nous semble cependant qu'un tel énoncé est naturel pour les raisons suivantes :

- Comme plusieurs auteurs l'ont déjà remarqué la décroissance des corrélations est liée à l'équirépartition des feuilles instables de $T$.

- Dire que les variétés instables sont bien réparties dans l'espace $\Omega$, c'est dire que l'intégrale d'une fonction $\psi$ sur un grand morceau de variété instable est proche de l'intégrale de $\psi$ sur $\Omega$. Pour cela il faut évidemment une certaine régularité de la fonction $\psi$. Mais quand on prend la moyenne d'une fonction $\psi$ le long d'une feuille instable, on régularise $\psi$ dans la direction instable. On peut donc s'attendre à ce que la régularité de la fonction $\psi$ le long de cette feuille ne soit pas importante quand on établit la propriété d'équirépartition.

Démonstration du lemme 1.3.1. — Soit un nombre réel $\eta>0$ et $f$ une fonction $\eta$-höldérienne. Soient des entiers naturels $a, b, c$ et $j, k, \ell, p, q, s$ vérifiant

$$
1 \leq a+b+c \leq 3 \text { et } 1 \leq j \leq k \leq \ell \leq \ell+p \leq \ell+q \leq \ell+s .
$$

TOME $133-2005-\mathrm{N}^{\mathrm{O}} 3$ 
Soit $F: \mathbb{R}^{4} \rightarrow \mathbb{R}$ une fonction différentiable. Comme la mesure de référence $\nu$ est invariante par $T$, nous avons

$$
\begin{aligned}
& \operatorname{Cov}\left(F\left(S_{j-1}, X_{j}, X_{k}, X_{\ell}\right), X_{\ell+p}{ }^{a} X_{\ell+q}{ }^{b} X_{\ell+s}{ }^{c}\right) \\
& \quad=\operatorname{Cov}\left(F\left(X_{1-\ell}+\cdots+X_{j-1-\ell}, X_{j-\ell}, X_{k-\ell}, X_{0}\right),\left(X_{0}^{a} X_{q-p}^{b} X_{s-p}^{c}\right) \circ T^{p}\right) .
\end{aligned}
$$

Posons

$$
\varphi:=F\left(X_{1-\ell}+\cdots+X_{j-1-\ell}, X_{j-\ell}, X_{k-\ell}, X_{0}\right) \quad \text { et } \quad \psi:=X_{0}^{a} X_{q-p}^{b} X_{s-p}^{c} .
$$

Pour tous $\bar{x} \in \Omega$ et $\bar{y} \in H_{u} \bar{x}$ tels que $d^{u}(\bar{x}, \bar{y}) \leq L_{0}$, grâce à la propriété de contraction (4), nous pouvons écrire

$$
\begin{aligned}
\left|\sum_{i=1}^{j-1} X_{-(k-i)}(\bar{x})-\sum_{i=1}^{j-1} X_{-(k-i)}(\bar{y})\right| & \leq \sum_{i=1}^{j-1} C_{f}^{(\eta, u)} d^{u}\left(T^{-(k-i)} \bar{x}, T^{-(k-i)}(\bar{y})\right)^{\eta} \\
& \leq \sum_{i=1}^{j-1} C_{f}^{(\eta, u)} C_{0}^{\eta} \delta^{\eta(k-i)} d^{u}(\bar{x}, \bar{y})^{\eta} \\
& \leq \frac{C_{f}^{(\eta, u)} C_{0}^{\eta}}{1-\delta^{\eta}} d^{u}(\bar{x}, \bar{y})^{\eta} \leq \frac{C_{f}^{(\eta, u)} C_{0}^{\eta}}{1-\delta^{\eta}} L_{0}^{\eta}
\end{aligned}
$$

et

$$
\begin{aligned}
\left|X_{-(k-j)}(\bar{x})-X_{-(k-j)}(\bar{y})\right| & \leq C_{f}^{(\eta, u)} d^{u}\left(T^{-(k-j)}(\bar{x}), T^{-(k-j)}(\bar{y})\right)^{\eta} \\
& \leq C_{f}^{(\eta, u)}\left(C_{0} d^{u}(\bar{x}, \bar{y})\right)^{\eta} \leq C_{f}^{(\eta, u)}\left(C_{0} L_{0}\right)^{\eta} .
\end{aligned}
$$

Notons $M$ la constante $C_{f}^{(\eta)} C_{0}^{\eta} /\left(1-\delta^{\eta}\right)$. En appliquant le théorème des accroissements finis, on obtient, pour tout $\bar{x} \in \Omega$ :

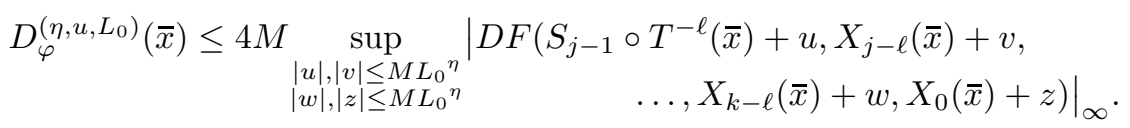

C'est pour obtenir une borne supérieure sur des boules de rayon borné que nous avons introduit la quantité $D_{\varphi}^{(\eta, u, L)}$. Pour tout $\bar{x} \in \Omega$ et tout $\bar{y} \in H_{s} H_{e} \bar{x}$, pour tout $i \in\{0, q-p, s-p\}$, nous avons

$$
\left|X_{i}(\bar{x})-X_{i}(\bar{y})\right| \leq C_{f}^{(\eta, s, e)}\left(d^{s, e}\left(T^{i}(\bar{x}), T^{i}(\bar{y})\right)\right)^{\eta} \leq C_{f}^{(\eta)}\left(C_{0} d^{s, e}(\bar{x}, \bar{y})\right)^{\eta},
$$

en utilisant la propriété de non-dilatation $(3)$; donc

$$
C_{X_{0}{ }^{a} X_{q-p}{ }^{b} X_{s-p^{c}}}^{(\eta, s, e)} \leq(a+b+c)\|f\|_{\infty}^{a+b+c-1} C_{f}^{(\eta)} C_{0}^{\eta} .
$$

Comme $X_{0}{ }^{a} X_{q-p}{ }^{b} X_{s-p}{ }^{c}$ est bornée par $\|f\|_{\infty}^{a+b+c}$, l'hypothèse du lemme appliquée à $\varphi$ et $\psi$ donne

$$
\begin{aligned}
& \operatorname{Cov}\left(F\left(S_{j-1}, X_{j}, X_{k}, X_{\ell}\right), X_{\ell+p}{ }^{a} X_{\ell+q}{ }^{b} X_{\ell+s}{ }^{c}\right) \\
& \leq C_{4} \alpha_{0}^{p}\left(\left\|F\left(S_{j-1}, X_{j}, X_{k}, X_{\ell}\right)\right\|_{1}\left(1+\|f\|_{\infty}^{3}+3\left(1+\|f\|_{\infty}^{2}\right) C_{f}^{(\eta)} C_{0}{ }^{\eta}\right)\right. \\
&\left.+\left(1+\|f\|_{\infty}^{3}\right)\left\|D_{\varphi}^{\left(\eta, u, L_{0}\right)}\right\|_{1}\right) .
\end{aligned}
$$

BULLETIN DE LA SOCIÉTÉ MATHÉMATIQUE DE FRANCE 
Nous allons exploiter ce lemme sous une forme légèrement modifiée. Étant donnés une tribu $\mathcal{A}$ et un entier $n$, notons $\mathcal{A}_{n}$ la tribu $\mathcal{A}_{n}:=T^{n} \mathcal{A}$.

Lemme 1.3.2. - S'il existe une tribu $\mathcal{A}$ et trois nombres réels $C_{5}>0, L_{1}$ et $\left.\alpha_{1} \in\right] 0,1[$ tels que, pour toutes fonctions $\varphi$ et $\psi$, et tout entier $n \geq 1$, nous ayons

$$
\left|\varphi-\mathbf{E}_{\nu}\left[\varphi \mid \mathcal{A}_{-n}\right]\right| \leq C_{5} D_{\varphi}^{\left(\eta, u, L_{1}\right)} \alpha_{1}^{n}
$$

et

$$
\left\|\mathbf{E}_{\nu}\left[\psi \mid \mathcal{A}_{n}\right]-\int_{\Omega} \psi \mathrm{d} \nu\right\|_{\infty} \leq C_{5}\left(\|\psi\|_{\infty}+C_{\psi}^{(\eta, s, e)}\right) \alpha_{1}{ }^{n}
$$

alors, pour toute fonction höldérienne $f$, le processus $\left(X_{k}\right)_{k \geq 0}=\left(f \circ T^{k}\right)_{k \geq 0}$ satisfait les hypothèses du théorème 2 .

Démonstration du lemme 1.3.2. - Une fonction $f$ est $\mathcal{A}_{n}$-mesurable si et seulement si $f \circ T^{n}$ est $\mathcal{A}_{0}$-mesurable. On a donc la relation suivante pour toute fonction $\nu$-intégrable $\varphi$ et pour tous entiers $n$ et $m$ :

$$
\mathbf{E}_{\nu}\left[\varphi \circ T^{m} \mid \mathcal{A}_{n}\right]=\mathbf{E}_{\nu}\left[\varphi \mid \mathcal{A}_{n+m}\right] \circ T^{m} .
$$

Considérons deux fonctions intégrables $\nu$-centrées $\varphi$ et $\psi$. Pour tout entier $n \geq 1$, nous avons (où $\lfloor$.$\rfloor désigne la partie entière) :$

$$
\begin{aligned}
& \left|\operatorname{Cov}_{\nu}\left(\varphi, \psi \circ T^{n}\right)\right|=\mathbf{E}_{\nu}\left[\varphi \cdot \psi \circ T^{n}\right] \\
& \leq \mathbf{E}_{\nu}\left[\mathbf{E}_{\nu}\left[\varphi \mid \mathcal{A}_{-\left\lfloor\frac{1}{2} n\right\rfloor}\right] \cdot \psi \circ T^{n}\right]+\mathbf{E}_{\nu}\left[\left(\varphi-\mathbf{E}_{\nu}\left[\varphi \mid \mathcal{A}_{-\left\lfloor\frac{1}{2} n\right\rfloor}\right]\right) \cdot \psi \circ T^{n}\right] \\
& \leq \mathbf{E}_{\nu}\left[\mathbf{E}_{\nu}\left[\varphi \mid \mathcal{A}_{-\left\lfloor\frac{1}{2} n\right\rfloor}\right] \cdot \psi \circ T^{n}\right]+C_{5}\left\|D_{\varphi}^{\left(\eta, u, L_{1}\right)}\right\|_{1} \alpha_{1}{ }^{\left\lfloor\frac{1}{2} n\right\rfloor}\|\psi\|_{\infty} \\
& \leq \mathbf{E}_{\nu}\left[\mathbf{E}_{\nu}\left[\varphi \mid \mathcal{A}_{-\left\lfloor\frac{1}{2} n\right\rfloor}\right] \cdot \mathbf{E}_{\nu}\left[\psi \circ T^{n} \mid \mathcal{A}_{-\left\lfloor\frac{1}{2} n\right\rfloor}\right]\right]+C_{5}\left\|D_{\varphi}^{\left(\eta, u, L_{1}\right)}\right\|_{1} \alpha_{1}{ }^{\left\lfloor\frac{1}{2} n\right\rfloor}\|\psi\|_{\infty} \\
& \leq \mathbf{E}_{\nu}\left[\mathbf{E}_{\nu}\left[\varphi \mid \mathcal{A}_{-\left\lfloor\frac{1}{2} n\right\rfloor}\right] \cdot \mathbf{E}_{\nu}\left[\psi \mid \mathcal{A}_{\left\lceil\frac{1}{2} n\right\rceil}\right] \circ T^{n}\right]+C_{5}\left\|D_{\varphi}^{\left(\eta, u, L_{1}\right)}\right\|_{1} \alpha_{1}\left\lfloor\frac{1}{2} n\right\rfloor\|\psi\|_{\infty} \\
& \leq\|\varphi\|_{L^{1}} C_{5}\left(\|\psi\|_{\infty}+C_{\psi}^{(\eta, s, e)}\right) \alpha_{1}^{\left\lceil\frac{1}{2} n\right\rceil}+C_{5}\left\|D_{\varphi}^{\left(\eta, u, L_{1}\right)}\right\|_{1} \alpha_{1}{ }^{\left\lfloor\frac{1}{2} n\right\rfloor}\|\psi\|_{\infty} .
\end{aligned}
$$

Il suffit maintenant d'appliquer le lemme précédent.

La tribu $\mathcal{A}_{0}$ sera la sous-tribu de $\mathcal{F}$ engendrée par une partition mesurable $\mathcal{P}$ de $\Omega$ dont les atomes sont des morceaux de variété instables. La première des inégalités ci-dessus est immédiate si $\psi$ est höldérienne dans la direction instable et si les atomes de $\mathcal{A}_{-n}$ sont de diamètres uniformément exponentiellement petits quand $n$ tend vers l'infini. La deuxième signifie que les feuilles instables sont bien réparties dans $\Omega$ (les atomes de $\mathcal{A}_{n}$ sont de «très grands » morceaux de feuilles instables quand $n$ tend vers l'infini).

TOME $133-2005-\mathrm{N}^{\mathrm{O}} 3$ 
1.4. Équirépartition. - On peut mettre en évidence un lien entre le mélange et l'équirépartition des variétés instables. Considérons un nombre réel $\varepsilon$ vérifiant $0<\varepsilon<r_{0}$ et un ensemble $F$ relativement compact inclus dans $H_{u}$. Appelons $U$ l'ensemble $B_{s}(\varepsilon) B_{e}(\varepsilon) F \bar{x}$. L'égalité

$$
T^{n} U=T^{n} B_{s}(\varepsilon) T^{-n} T^{n} B_{e}(\varepsilon) T^{-n} T^{n} F T^{-n} T^{n} \bar{x}
$$

montre que, sous l'action de $T^{n}$, l'ensemble $U$ est contracté dans la direction $H_{s}$, dilaté dans la direction $H_{u}$, inchangé dans la direction $H_{e}$. Notons $\mathcal{V}_{n}$ l'ensemble

$$
\mathcal{V}_{n}:=T^{n} B_{s}(\varepsilon) T^{-n} \times T^{n} B_{e}(\varepsilon) T^{-n} \times T^{n} F T^{-n} .
$$

Soit $\varphi$ une fonction $\eta$-höldérienne sur $G / \Gamma$. L'expression de la mesure $\bar{\mu}$ dans la décomposition de Bruhat donne la forme suivante au produit scalaire $\left\langle 1_{U} \circ T^{-n}, \varphi\right\rangle$ :

$$
\left\langle 1_{U} \circ T^{-n}, \varphi\right\rangle=\int_{\mathcal{V}_{n}} \Theta_{e}\left(h_{e}\right) \varphi\left(h_{s} h_{e} h_{u} T^{n} \bar{x}\right) \mathrm{d} h_{s} \mathrm{~d} h_{e} \mathrm{~d} h_{u} .
$$

Le diamètre de $T^{n} B_{s}(\varepsilon) T^{-n} \times T^{n} B_{e}(\varepsilon) T^{-n}$ est inférieur à $2 \varepsilon$. Nous avons donc, pour tout $\left(h_{s}, h_{e}, h_{u}\right) \in \mathcal{V}_{n}$ :

$$
\left|\varphi\left(h_{s} h_{e} h_{u} T^{n} \bar{x}\right)-\varphi\left(h_{u} T^{n} \bar{x}\right)\right| \leq 2^{\eta} C_{\varphi}^{(\eta)} \varepsilon^{\eta}
$$

et par conséquent

$$
\left|\left\langle 1_{U} \circ T^{-n}, \varphi\right\rangle-\int_{\mathcal{V}_{n}} \Theta_{e}\left(h_{e}\right) \varphi\left(h_{u} T^{n} \bar{x}\right) \mathrm{d} h_{s} \mathrm{~d} h_{e} \mathrm{~d} h_{u}\right| \leq 2^{\eta} C_{\varphi}^{(\eta)} \varepsilon^{\eta} \bar{\mu}(U) .
$$

Notons $\mu_{u}$ la mesure de Haar sur $H_{u}$ et $d_{e}+d_{s}$ la dimension de $H_{s} H_{e}$. En divisant par $\bar{\mu}(U)$ qui est à peu près égal à $\varepsilon^{d_{e}+d_{s}} \mu_{u}(F)$, nous obtenons la majoration

(9) $\left|\frac{1}{\mu_{u}\left(T^{n} F T^{-n}\right)} \int_{T^{n} F T^{-n}} \varphi\left(h_{u} T^{n} \bar{x}\right) \mathrm{d} h_{u}\right| \leq C_{6}\left(\frac{\left\langle 1_{U} \circ T^{-n}, \varphi\right\rangle}{\varepsilon^{d_{e}+d_{s}} \mu_{u}(F)}+2^{\eta} C_{\varphi}^{(\eta)} \varepsilon^{\eta}\right)$

sur laquelle repose la démonstration de la proposition suivante.

Pour tout nombre réel $\beta>0$ et tout sous-ensemble $F$ de $H_{u}$, nous notons $\partial F(\beta)$ l'ensemble des points de $H_{u}$ qui sont à une distance inférieure à $\beta$ du bord de $F$ et $\partial U(\beta)$ l'ensemble des points de $G / \Gamma$ qui sont à une distance inférieure à $\beta$ du bord de $U$.

Proposition 1.3. - Soit $F \subseteq H_{u}$ un ensemble de diamètre inférieur à $r_{0}$ tel qu'il existe deux nombres réels $B>0$ et $\alpha>0$ tels que, pour tout $\beta>0$, on ait

$$
\mu_{u}(\partial F(\beta)) \leq B \beta^{\alpha} .
$$

Alors, il existe deux nombres réels $K_{0}>0$ et $\xi_{0}>1$ tels que, pour toute fonction intégrable $\bar{\mu}$-centrée $\varphi$ sur $G / \Gamma$, pour tout entier $n \geq 1$, pour tout $\bar{x} \in G / \Gamma$, on ait

$$
\left|\frac{1}{\mu_{u}\left(T^{n} F T^{-n}\right)} \int_{T^{n} F T^{-n}} \varphi\left(h_{u} \bar{x}\right) \mathrm{d} h_{u}\right| \leq \frac{K_{0}}{\mu_{u}(F)}\left(\|\varphi\|_{\infty}+C_{\varphi}^{(\eta)}\right) \xi_{0}{ }^{-n} .
$$

BULletin DE LA SOCiÉtÉ MATHÉmATiQUe DE FRANCE 
Démonstration de la proposition 1.3. - L'inégalité de mélange (5) appliquée à $\chi_{n}^{(\rho)} * 1_{U}$ et à $\chi_{n}^{(\rho)} * \varphi$ et les propriétés de la suite $\chi_{n}^{(\rho)}$ assurent l'existence d'une constante $C_{7}>0$ telle que

$$
\begin{aligned}
\left|\left\langle\left(\chi_{n}^{(\rho)} * 1_{U}\right) \circ T^{-n}, \chi_{n}^{(\rho)} * \varphi\right\rangle\right| & \leq C_{3}\left\|\Delta \chi_{n}^{(\rho)} * 1_{U}\right\|_{2} \cdot\left\|\Delta\left(\chi_{n}^{(\rho)} * \varphi\right)\right\|_{2} \zeta^{-n} \\
& \leq C_{7}\|\varphi\|_{\infty} \rho^{2 C_{2} n} \zeta^{-n} .
\end{aligned}
$$

On a aussi

$\left|\left\langle 1_{U} \circ T^{-n}, \varphi\right\rangle-\left\langle\left(\chi_{n}^{(\rho)} * 1_{U}\right) \circ T^{-n}, \chi_{n}^{(\rho)} * \varphi\right\rangle\right| \leq \rho^{-n \eta} C_{\varphi}^{(\eta)}+\|\varphi\|_{\infty} \bar{\mu}\left(\partial U\left(\rho^{-n}\right)\right)$.

Compte tenu de (9), ces inégalités fournissent la majoration

$$
\begin{aligned}
& \left|\frac{1}{\mu_{u}\left(T^{n} F T^{-n}\right)} \int_{T^{n} F T^{-n}} \varphi\left(h_{u} T^{n} \bar{x}\right) \mathrm{d} h_{u}\right| \\
& \quad \leq C_{6}\left(\frac{\rho^{-n \eta} C_{\varphi}^{(\eta)}+\|\varphi\|_{\infty} \bar{\mu}\left(\partial U\left(\rho^{-n}\right)\right)+C_{7} \rho^{2 C_{2} n} \zeta^{-n}}{\varepsilon^{d_{e}+d_{s}} \mu_{u}(F)}+2 C_{\varphi}^{(\eta)} \varepsilon^{\eta}\right) .
\end{aligned}
$$

Il suffit maintenant de bien choisir $\rho>1$ et de faire dépendre $\varepsilon$ convenablement de $n$ pour obtenir la proposition.

Nous allons maintenant voir qu'on peut obtenir une estimation analogue à celle de la proposition 1.3 faisant intervenir uniquement la régularité de $\varphi$ dans la direction $H_{e} H_{s}$.

Proposition 1.4. - Soit $F \subseteq H_{u}$ un ensemble de diamètre inférieur à $r_{0}$ tel qu'il existe $B>0$ et $\alpha$ tels que, pour tout $\beta>0$, on ait

$$
\mu_{u}(\partial F(\beta)) \leq B \beta^{\alpha}
$$

Il existe $\xi_{1}>1$ et $K_{1}>0$ tels que, pour toute fonction intégrable $\varphi \bar{\mu}$-centrée, pour tout entier $n \geq 1$ et pour tout $\bar{x} \in G / \Gamma$, on ait

$$
\left|\frac{1}{\mu_{u}\left(T^{n} F T^{-n}\right)} \int_{T^{n} F T^{-n}} \varphi\left(h_{u} \bar{x}\right) \mathrm{d} h_{u}\right| \leq \frac{K_{1}}{\mu_{u}(F)}\left(\|\varphi\|_{\infty}+C_{\varphi}^{(\eta, s, e)}\right) \xi_{1}^{-n} .
$$

Démonstration de la proposition 1.4. - Considérons une fonction $\varphi$ à support dans un ensemble $P=B_{u}\left(r_{0}\right) B_{e}\left(r_{0}\right) B_{s}\left(r_{0}\right) \bar{x}$. Pour régulariser $\varphi$ dans la direction $H_{u}$, nous allons l'intégrer dans cette direction. Soit $g$ une fonction $C^{\infty}$ à support dans $B_{u}\left(r_{0}\right)$, positive, d'intégrale $1\left(C_{g}^{(1)}\right.$ désignera le coefficient de Lipschitz de $g$ ). Définissons la fonction $\psi$ par

(a) $\psi\left(h_{u} h_{e} h_{s} \bar{x}\right)=g\left(h_{u}\right) \int_{B_{u}\left(r_{0}\right)} \varphi\left(h_{u}^{\prime} h_{e} h_{s} \bar{x}\right) \mathrm{d} h_{u}^{\prime}$ si $\left(h_{u}, h_{e}, h_{s}\right)$ appartient à $B_{u}\left(r_{0}\right) \times B_{e}\left(r_{0}\right) \times B_{s}\left(r_{0}\right)$,

(b) $\psi(\bar{y})=0$ si $\bar{y}$ n'appartient pas à $P$.

La constante $C_{1}$ apparaissant dans le lemme suivant a été définie dans le paragraphe intitulé « Régularité de l'holonomie le long des feuilles $H_{e} H_{s}$ ».

TOME $133-2005-\mathrm{N}^{\mathrm{O}} 3$ 
LEMME 1.5. - Les fonctions $\varphi$ et $\psi$ ont la même intégrale et il existe $K_{2}>0$ indépendant de $\varphi$ tel que,

$$
C_{\psi}^{(\eta)} \leq K_{2}\left(\|\varphi\|_{\infty}+C_{\varphi}^{(\eta, s, e)}\right) .
$$

Démonstration du lemme 1.5. - On vérifie le premier point en utilisant le théorème de Fubini. Montrons l'inégalité. Soient $\left(h_{u}, h_{e}, h_{s}\right)$ et $\left(h_{u}^{\prime}, h_{e}^{\prime}, h_{s}^{\prime}\right)$ deux points de $B_{u}\left(r_{0}\right) \times B_{e}\left(r_{0}\right) \times B_{s}\left(r_{0}\right)$. On a

$$
\begin{aligned}
& \left|\psi\left(h_{u} h_{e} h_{s} \bar{x}\right)-\psi\left(h_{u}^{\prime} h_{e}^{\prime} h_{s}^{\prime} \bar{x}\right)\right| \\
& =\left|g\left(h_{u}\right) \int_{B_{u}\left(2 r_{0}\right)} \varphi\left(\widetilde{h}_{u} h_{e} h_{s} \bar{x}\right) \mathrm{d} \widetilde{h}_{u}-g\left(h_{u}^{\prime}\right) \int_{B_{u}\left(r_{0}\right)} \varphi\left(\widetilde{h}_{u}^{\prime} h_{e}^{\prime} h_{s}^{\prime} \bar{x}\right) \mathrm{d} \widetilde{h}_{u}^{\prime}\right| \\
& \leq\|\varphi\|_{\infty} \cdot\left|g\left(h_{u}\right)-g\left(h_{u}^{\prime}\right)\right| \mu_{u}\left(B_{u}\left(r_{0}\right)\right) \\
& \quad+\|g\|_{\infty} \cdot\left|\int_{B_{u}\left(r_{0}\right)} \varphi\left(\widetilde{h}_{u} h_{e} h_{s} \bar{x}\right) \mathrm{d} \widetilde{h}_{u}^{\prime}-\int_{B_{u}\left(r_{0}\right)} \varphi\left(\widetilde{h}_{u}^{\prime} h_{e}^{\prime} h_{s}^{\prime} \bar{x}\right) \mathrm{d} \widetilde{h}_{u}^{\prime}\right| .
\end{aligned}
$$

Les propriétés de l'application d'holonomie rappelées plus haut nous autorisent à écrire

$$
\begin{aligned}
& \quad\left|\int_{B_{u}\left(r_{0}\right)} \varphi\left(\widetilde{h}_{u} h_{e} h_{s} \bar{x}\right) \mathrm{d} \widetilde{h}_{u}-\int_{B_{u}\left(r_{0}\right)} \varphi\left(\widetilde{h}_{u}^{\prime} h_{e}^{\prime} h_{s}^{\prime} \bar{x}\right) \mathrm{d} \widetilde{h}_{u}^{\prime}\right| \\
& =\left|\int_{B_{u}\left(r_{0}\right)} \varphi\left(\widetilde{h}_{u} h_{e} h_{s} \bar{x}\right) \mathrm{d} \widetilde{h}_{u}-\int_{B_{u}\left(2 r_{0}\right)} \varphi\left(\mathcal{H}\left(\widetilde{h}_{u} h_{e} h_{s} \bar{x}\right)\right) \frac{\mathrm{d} \mathcal{H}\left(\mu_{u}\right)}{\mathrm{d} \mu_{u}}\left(\widetilde{h}_{u} h_{e} h_{s} \bar{x}\right) \mathrm{d} \widetilde{h}_{u}\right| \\
& \leq\left|\int_{B_{u}\left(2 r_{0}\right)}\left(\varphi\left(\widetilde{h}_{u} h_{e} h_{s} \bar{x}\right)-\varphi\left(\mathcal{H}\left(\widetilde{h}_{u} h_{e} h_{s} \bar{x}\right)\right)\right) \mathrm{d} \widetilde{h}_{u}\right| \\
& \quad+\int_{B_{u}\left(2 r_{0}\right)}\left|\varphi\left(\mathcal{H}\left(\widetilde{h}_{u} h_{e} h_{s} \bar{x}\right)\right)\right| \cdot\left|\frac{\mathrm{d} \mathcal{H}\left(\mu_{u}\right)}{\mathrm{d} \mu_{u}}\left(\widetilde{h}_{u} h_{e} h_{s} \bar{x}\right)-1\right| \mathrm{d} \widetilde{h}_{u} \\
& \leq \mu_{u}\left(B_{u}\left(2 r_{0}\right)\right) C_{1} C_{\varphi}^{(\eta, s, e)} d\left(h_{e} h_{s}, h_{e}^{\prime} h_{s}^{\prime}\right)^{\eta}+C_{1}\|\varphi\|_{\infty} \mu_{u}\left(B_{u}\left(2 r_{0}\right)\right) d\left(h_{e} h_{s}, h_{e}^{\prime} h_{s}^{\prime}\right) \\
& \leq \mu_{u}\left(B_{u}\left(2 r_{0}\right)\right) C_{1}\left(\|\varphi\|_{\infty}+C_{\varphi}^{(\eta, s, e)}\right) d\left(h_{e} h_{s}, h_{e}^{\prime} h_{s}^{\prime}\right)^{\eta} .
\end{aligned}
$$

On obtient donc

$$
\begin{aligned}
\left|\psi\left(h_{u} h_{e} h_{s} \bar{x}\right)-\psi\left(h_{u}^{\prime} h_{e}^{\prime} h_{s}^{\prime} \bar{x}\right)\right| & \\
\leq & \|g\|_{\infty} \mu_{u}\left(B_{u}\left(2 r_{0}\right)\right) C_{1}\left(\|\varphi\|_{\infty}+C_{\varphi}^{(\eta, s, e)}\right) d\left(h_{e} h_{s}, h_{e}^{\prime} h_{s}^{\prime}\right)^{\eta} \\
& +C_{g}^{(1)} \mu_{u}\left(B_{u}\left(r_{0}\right)\right)\|\varphi\|_{\infty} d\left(h_{u}, h_{u}^{\prime}\right) .
\end{aligned}
$$

Suite de la démonstration de la proposition 1.4. - La fonction $\psi$ étant $\eta$-höldérienne, nous pouvons lui appliquer la proposition 1.3. Pour tout entier $n \geq 1$, tout $\bar{x}$, nous avons

$$
\left|\frac{1}{\mu_{u}\left(T^{n} F T^{-n}\right)} \int_{T^{n} F T^{-n}} \psi\left(h_{u} \bar{x}\right) \mathrm{d} h_{u}\right| \leq \frac{K_{0}\left(C_{\psi}^{(\eta, s, e)}+\|\psi\|_{\infty}\right) \xi_{0}^{-n}}{\mu_{u}(F)} .
$$

BULLETIN DE LA SOCIÉTÉ MATHÉMATIQUE DE FRANCE 
Il nous reste à estimer la différence entre les intégrales

$$
\frac{1}{\mu_{u}\left(T^{n} F T^{-n}\right)} \int_{T^{n} F T^{-n}} \psi\left(h_{u} \bar{x}\right) \mathrm{d} h_{u} \text { et } \frac{1}{\mu_{u}\left(T^{n} F T^{-n}\right)} \int_{T^{n} F T^{-n}} \varphi\left(h_{u} \bar{x}\right) \mathrm{d} h_{u} .
$$

Celles-ci sont des sommes d'intégrales de $\varphi$ et $\psi$ sur les composantes connexes des intersections de $P=B_{u}\left(r_{0}\right) B_{e}\left(r_{0}\right) B_{s}\left(r_{0}\right) \bar{x}$ avec $T^{n} F T^{-n}$. Elles ne diffèrent éventuellement que sur les morceaux contenant un point du bord de $T^{n} F T^{-n} \bar{x}$. Nous avons donc

$$
\left|\frac{1}{\mu_{u}\left(T^{n} F T^{-n}\right)} \int_{T^{n} F T^{-n}}\left(\psi\left(h_{u} \bar{x}\right)-\varphi\left(h_{u} \bar{x}\right)\right) \mathrm{d} h_{u}\right| \leq 2\|\varphi\|_{\infty} \frac{\mu_{u}\left(\partial T^{n} F T^{-n}\left(r_{0}\right)\right)}{\mu_{u}\left(T^{n} F T^{-n}\right)} .
$$

D'après la propriété de contraction (4), pour tout $n \geq 1$ et tout couple $\left(h_{u}, h_{u}^{\prime}\right) \in H_{u}{ }^{2}$, on a

$$
d_{0}\left(T^{-n} h_{u} T^{n}, T^{-n} h_{u}^{\prime} T^{n}\right) \leq C_{0} d_{0}\left(h_{u}, h_{u}^{\prime}\right) \delta^{-n} .
$$

Soit $h_{u}$ un point de $T^{n} F T^{-n}$ à une distance inférieure à $r_{0}$ du bord de $T^{n} F T^{-n}$; il existe $h_{u}^{\prime} \in \partial T^{n} F T^{-n}$ tel que

$$
d_{0}\left(h_{u}, h_{u}^{\prime}\right) \leq r_{0} .
$$

On a alors $d_{0}\left(T^{-n} h_{u} T^{n}, T^{-n} h_{u}^{\prime} T^{n}\right) \leq C r_{0} \delta^{-n} ;$ autrement $\operatorname{dit} T^{-n} h_{u} T^{n}$ appartient à $\partial F\left(C r_{0} \delta^{-n}\right)$. On en déduit que $\partial T^{n} F T^{-n}\left(r_{0}\right)$ est inclus dans $T^{n} \partial F\left(C r_{0} \delta^{-n}\right) T^{-n}$. L'action de $T$ sur $H_{u}$ par conjugaison est linéaire. On a donc

$$
\frac{\mu_{u}\left(\partial T^{n} F T^{-n}\left(r_{0}\right)\right)}{\mu_{u}\left(T^{n} F T^{-n}\right)} \leq \frac{\mu_{u}\left(T^{n} \partial F\left(C r_{0} \delta^{-n}\right) T^{-n}\right)}{\mu_{u}\left(T^{n} F T^{-n}\right)} \leq \frac{\mu_{u}\left(\partial F\left(C r_{0} \delta^{-n}\right)\right)}{\mu_{u}(F)} .
$$

Pour terminer la démonstration, grâce à une partition de l'unité liée à un recouvrement fini de $G / \Gamma$ par des ensembles de la forme $B_{u}\left(r_{0}\right) B_{e}\left(r_{0}\right) B_{s}\left(r_{0}\right) \bar{y}$, on s'affranchit de la condition sur le support de $\varphi$.

1.5. Fin de la démonstration du théorème 3. - À partir d'un recouvrement $\mathcal{R}$ fini de $G / \Gamma$ par des boîtes de la forme $B_{u}\left(r_{0}\right) B_{e}\left(r_{0}\right) B_{s}\left(r_{0}\right) \bar{y}$, nous construisons une partition $\mathcal{P}$ (non dénombrable) en morceaux de feuilles dilatées. On définit la partition $\mathcal{Q}$ de $G / \Gamma$ par

$$
\mathcal{Q}=\left\{\bigcap_{R \in \mathcal{R}} A_{R} ; A_{R}=R \text { ou }{ }^{c} R\right\},
$$

puis la partition $\mathcal{P}$ par

$$
\mathcal{P}(\bar{x})=\mathcal{Q}(\bar{x}) \cap B_{u}\left(3 r_{0}\right) \bar{x},
$$

Nous notons $\mathcal{A}$ la tribu engendrée. Les atomes de $\mathcal{A}$ sont de la forme $F \bar{x}$ où les ensembles $F$ sont des parties relativement compactes de $H_{u}$ dont le bord est régulier. Nous supposons qu'il existe un nombre réel $\alpha>0$ tel que pour tout atome $F \bar{x}$ de $\mathcal{A}$ on ait $\mu_{u}(F)>\alpha$ (on peut toujours s'y ramener). Notons $\mathcal{A}_{n}$ la tribu $T^{n} \mathcal{A}$. Soient $\varphi$ et $\psi$ deux fonctions d'intégrales nulles sur $G / \Gamma$ et un 
entier $n \geq 1$. Les atomes de $\mathcal{A}_{-n}$ sont des ensembles de la forme $T^{-n} F T^{n} T^{-n} \bar{x}$. Les diamètres des atomes de $\mathcal{A}_{-n}$ sont donc inférieurs à $C_{0} \delta^{-n}$ et on a

$$
\left|\varphi-\mathbf{E}_{\bar{\mu}}\left[\varphi \mid \mathcal{A}_{-n}\right]\right| \leq C_{0}^{\eta} D_{\varphi}^{\left(\eta, u, 3 r_{0}\right)} \delta^{-\eta n} .
$$

Les valeurs de $\mathbf{E}_{\bar{\mu}}\left[\psi \mid \mathcal{A}_{n}\right]$ sont données par les moyennes de $\psi$ sur les atomes de $\mathcal{A}_{n}$. On peut appliquer la proposition 1.4. Vues les propriétés des atomes de $\mathcal{A}$, on obtient l'existence d'une constante $K_{3}$ telle que

$$
\left\|\mathbf{E}_{\bar{\mu}}\left[\psi \mid \mathcal{A}_{n}\right]\right\|_{\infty} \leq K_{3}\left(\|\psi\|_{\infty}+C_{\psi}^{(\eta, s, e)}\right) \xi_{1}^{-n}
$$

Comme le montre le lemme 1.3.2, les inégalités (10) et (11) donnent le résultat.

\section{L'exemple de Shub et Wilkinson}

2.1. Énoncé du résultat. — Considérons l'automorphisme algébrique $T_{0}$ du tore $\mathbb{T}^{3}$ défini par la matrice

$$
T_{0}=\left(\begin{array}{lll}
2 & 1 & 0 \\
1 & 1 & 0 \\
0 & 0 & 1
\end{array}\right) .
$$

Shub et Wilkinson ont montré qu'il existe $T$, une perturbation $C^{2}$ de $T_{0}$, ayant les propriété suivantes :

- $T$ est un difféomorphisme ergodique de $\mathbb{T}^{3}$ préservant la mesure de Lebesgue $\nu$;

- T est non uniformément hyperbolique; il existe trois sous-fibrés continus invariants par $T: E^{s}$ (dit stable), $E^{c}$ (dit central) et $E^{u}$ (dit instable) de $\mathcal{T} \mathbb{T}^{3}$, de dimension 1 , en somme directe, et des nombres réels $\lambda_{1}, \lambda_{2}$, $\lambda_{3}, \lambda_{4}, \lambda_{5}$ et $\lambda_{6}$ tels que

$$
\begin{gathered}
\mathrm{e}^{\lambda_{1}} \leq\left(\left(D_{x} T\right)_{\mid E^{s}(x)}\right) \leq \mathrm{e}^{\lambda_{2}}<\mathrm{e}^{\lambda_{3}} \leq\left(\left(D_{x} T\right)_{\mid E^{s}(c)}\right) \leq \mathrm{e}^{\lambda_{4}}, \\
\mathrm{e}^{\lambda_{4}}<\mathrm{e}^{\lambda_{5}} \leq\left(\left(D_{x} T\right)_{\mid E^{s}(x)}\right) \leq \mathrm{e}^{\lambda_{6}},
\end{gathered}
$$

avec $\lambda_{2}<0$ et $\lambda_{5}>0$;

- il existe trois nombres réels $\lambda^{s}<\lambda^{c}<0<\lambda^{u}$ (les exposants de Lyapounov de $T$ ), tels que, pour tout $i=s, c, u$, pour presque tout point $x$, pour tout vecteur $v$ appartenant à $E^{i}(x)$, on ait

$$
\lim _{n \rightarrow+\infty} \frac{1}{n} \log \left\|D_{x} T^{n} v\right\|=\lambda^{i}
$$

- le sous-fibré $E^{c}$ est intégrable et définit une fibration en cercle de $\mathbb{T}^{3}$ non absolument continue.

Notons que l'énoncé de Shub et Wilkinson est légèrement différent : $\lambda^{c}$ est positif dans [29] (il suffit de changer $T$ en $T^{-1}$ pour retrouver leur formulation). Remarquons d'ailleurs que lorsqu'on s'intéresse au théorème limite central pour une transformation $T$ inversible, comme c'est le cas ici, il est sans importance d'étudier $T$ ou $T^{-1}$. Nous avons choisi de présenter leur résultat de cette façon 
car nous utiliserons des résultats de Dolgopyat sur les transformations partiellement hyperboliques dont la direction centrale est asymptotiquement contractée : il a montré dans [8] qu'on peut contrôler en mesure le défaut d'uniformité du système $\left(\mathbb{T}^{3}, T, \nu\right)$.

THÉORÈme 4. - Soit $\left(\mathbb{T}^{3}, \nu, T\right)$ comme ci-dessus et $f: \mathbb{T}^{3} \rightarrow \mathbb{R}$ une fonction höldérienne, $\nu$-centrée. Alors, la limite suivante existe :

$$
\sigma:=\lim _{n \rightarrow+\infty}\left(\mathbf{E}_{\nu}\left[\left(\frac{1}{\sqrt{n}} \sum_{k=1}^{n} f \circ T^{k}\right)^{2}\right]\right)^{\frac{1}{2}} .
$$

- Si $\sigma=0$, alors la suite de v.a. $\left(\sum_{k=1}^{n} f \circ T^{k}\right)_{n \geq 1}$ est bornée dans $L^{2}$.

- Si $\sigma>0$, alors la suite de variables aléatoires $\left(1 / \sqrt{n} \sum_{k=1}^{n} f \circ T^{k}\right)_{n>0}$ converge en loi relativement à la mesure de probabilité $\nu$ (lorsque $n$ tend vers $+\infty)$ vers une variable aléatoire $N$ de loi normale centrée de variance $\sigma^{2}$ et il existe un nombre réel $R>0$ tel que, pour tout nombre entier $n \geq 1$, on ait

$$
\sup _{x \in \mathbb{R}}\left|\nu\left(\frac{1}{\sqrt{n}} \sum_{k=1}^{n} f \circ T^{k} \leq x\right)-\mathbf{P}(N \leq x)\right| \leq \frac{R}{\sqrt{n}} .
$$

2.2. Quelques notations et résultats de Dolgopyat. — Le sous-fibré $E^{u}$ est intégrable et définit un feuilletage $\mathcal{F}^{u}$ dit feuilletage en feuilles instables.

Suivant [8], nous introduisons l'ensemble $\mathcal{V}$ des courbes instables (i.e. des courbes contenues dans un élément de $\mathcal{F}^{u}$ ) de longueur comprise entre 1 et 2 .

Introduisons une mesure de probabilité $\ell_{V}$ sur chaque courbe instable bornée $V$ comme suit. Soit une courbe instable $V$ de longueur finie; pour tous éléments $z$ et $z^{\prime}$ de $V$, nous définissons

$$
\widetilde{\rho}_{V}\left(z, z^{\prime}\right):=\prod_{j=0}^{+\infty} \frac{\left(\mathrm{d} T^{-1} \mid E^{u}\right)\left(T^{-j}(z)\right)}{\left(\mathrm{d} T^{-1} \mid E^{u}\right)\left(T^{-j}\left(z^{\prime}\right)\right)} .
$$

En raison des contrôles sur $\left(\mathrm{d} T \mid E^{u}\right)$ donnés par la propriété d'hyperbolicité partielle, il existe une constante $\widetilde{C}_{0}>1$ telle qu'on ait $\widetilde{C}_{0}^{-1} \leq \widetilde{\rho}_{V}(.,.) \leq \widetilde{C}_{0}$. Nous considérons la mesure de probabilité $\ell_{V}$ sur $V$ donnée par

$$
\mathrm{d} \ell_{V}(z)=\rho_{V}(z) \mathrm{d} z, \quad \text { avec } \quad \rho_{V}(z):=\frac{1}{\int_{V} \widetilde{\rho}_{V}\left(z^{\prime}, z_{0}\right) \mathrm{d} z^{\prime}} \widetilde{\rho}_{V}\left(z, z_{0}\right),
$$

$z_{0}$ étant un point de $V$ fixé (remarquons que la définition de $\rho_{V}$ ne dépend pas du point $z_{0}$ choisi dans $V$ ). Par définition, la mesure image de $\ell_{V}$ par $T$ est la mesure $\ell_{T(V)}$. Nous reprenons les notations de [8] : nous notons $E_{1}(0)$ l'ensemble des mesures de probabilités $\ell_{V}$ avec $V \in \mathcal{V}$; nous notons $E_{2}(0)$ l'enveloppe convexe de $E_{1}(0)$ et $E(0)$ l'adhérence de $E_{2}(0)$ dans l'ensemble des mesures de probabilité sur $\Omega$.

Un résultat essentiel pour notre démonstration est le lemme technique 6.1 de [8] que nous reformulons ci-dessous (ayant remarqué que les constantes y

TOME $133-2005-\mathrm{N}^{\mathrm{O}} 3$ 
apparaissant sont uniformes). Pour toute courbe instable $V$ de longueur finie, nous notons $m_{V}$ la mesure de probabilité sur $V \times[0,1]$ donnée par $\mathrm{d} m_{V}(x, t)=$ $\mathrm{d} \ell_{V}(x) \mathrm{d} t$.

Proposition 2.1. - Il existe des nombres réels $\left.C_{1}>0, C_{2}>0, \rho_{1} \in\right] 0,1[$ et $\left.\rho_{2} \in\right] 0,1[$ et deux familles de fonctions mesurables

$$
\begin{aligned}
& \left\{\tau_{V, V^{\prime}}: V \times[0,1] \rightarrow V^{\prime} \times[0,1]\right\}_{\left(V, V^{\prime}\right) \in \mathcal{V} \times \mathcal{V}}, \\
& \left\{R_{V, V^{\prime}}: V \times[0,1] \rightarrow \mathbb{N}\right\}_{\left(V, V^{\prime}\right) \in \mathcal{V} \times \mathcal{V}}
\end{aligned}
$$

tels que, pour tous $\left(V, V^{\prime}\right) \in \mathcal{V} \times \mathcal{V}$, tout $(x, t) \in V \times[0,1]$ et tout $\left(x^{\prime}, t^{\prime}\right) \in$ $V^{\prime} \times[0,1]$, nous avons :

(a) la mesure image de $m_{V}$ par $\tau_{V, V^{\prime}}$ est $m_{V^{\prime}}$;

(b) si $\left(x^{\prime}, t^{\prime}\right)=\tau_{V, V^{\prime}}(x, t)$, alors, pour tout entier naturel $n$, nous avons

$$
d\left(T^{n}(x), T^{n}\left(x^{\prime}\right)\right) \leq C_{1} \rho_{1}{ }^{n-R_{V, V^{\prime}}(x, t)} ;
$$

(c) pour tout entier naturel $N$, nous avons $m_{V}\left(\left\{R_{V, V^{\prime}} \geq N\right\}\right) \leq C_{2} \rho_{2}{ }^{N}$.

Dolgopyat [8] utilise ce résultat pour établir le résultat d'équirépartition suivant pour la mesure $\nu$

Corollaire 2.2 (corollaire 6.3 de [8]). — La mesure $\nu$ est l'unique mesure $T$-invariante appartenant à $E(0)$. De plus, il existe deux nombres réels $C_{3}>0$ et $\left.\rho_{3} \in\right] 0,1[$ tels que, pour tout $\ell$ dans $E(0)$, toute fonction $\eta$-höldérienne $A: \mathbb{T}^{3} \rightarrow \mathbb{C}$ et tout entier naturel $n$, nous avons :

$$
\left|\ell\left(A \circ T^{n}\right)-\nu(A)\right| \leq C_{3}\left(\|A\|_{\infty}+C_{A}^{(\eta)}\right) \rho_{3}{ }^{n} .
$$

Il suffit de modifier un peu la démonstration de [8] pour démontrer le théorème 4 .

2.3. Démonstration du théorème 4. - Plaçons-nous sous les hypothèses $\mathrm{du}$ théorème 4 . Supposons que la fonction $f: \mathbb{T}^{3} \rightarrow \mathbb{R}$ est $\eta$-höldérienne, avec $\eta \in] 0 ; 1]$. Nous ne pouvons pas utiliser directement l'argument exposé dans le paragraphe précédent : il n'y a pas de raison pour que la condition de non-dilatation (3) soit vérifiée. Cependant, la démonstration du théorème 4 repose sur une idée analogue : nous utiliserons le fait que la propriété (3) est vérifiée pour $n$ assez grand sur un ensemble de grande mesure (cf. proposition 2.1 ci-dessous).

- Sous l'action de $T^{-1}$, le diamètre de $V$ est contracté avec vitesse exponentielle. Il existe donc deux nombres réels $C_{4}>0$ et $\left.\rho_{4} \in\right] 0,1[$ tels que pour tout $V^{\prime}$ dans $\mathcal{V}$ et tout entier naturel $N$, le diamètre de $T^{-N}\left(V^{\prime}\right)$ est majoré par $C_{4} \rho_{4}{ }^{N}$. Dans la suite, nous considérons un tel couple $\left(C_{4}, \rho_{4}\right)$ de nombres réels. Soient $C_{1}, \rho_{1}, C_{2}, \rho_{2}$ des nombres réels pour lesquels l'énoncé de la proposition 2.1 est valable. 
- Soit un nombre réel $\eta>0$. Soit $f$ une fonction $\eta$-höldérienne $\nu$-centrée. Pour tout entier $k$, nous notons $X_{k}:=f \circ T^{k}$. Nous allons montrer que $\left(X_{k}\right)_{k}$ vérifie l'hypothèse du théorème 2 avec

$$
\begin{aligned}
& M:=\max \left\{1,\|f\|_{\infty}, C_{f}^{(\eta)} \frac{C_{4}^{\eta}}{1-\rho_{4} \eta}\right\}, \\
& C:=3 M\left(1+\|f\|_{\infty}^{3}\right)+\left(2+3\|f\|_{\infty}^{3}\right)\left(C_{f}^{(\eta)} \frac{C_{1}^{\eta}}{\rho_{1}^{\frac{1}{2} \eta}}+\frac{C_{2}}{\rho_{2}^{\frac{3}{4}}}\right), \\
& \xi_{p, \ell}=\max \left\{\rho_{4}^{\frac{1}{2} p \eta}, \rho_{1}^{\frac{1}{4} p \eta}, \rho_{2}^{\frac{1}{4} p}\right\} .
\end{aligned}
$$

Pour cela, nous utilisons la proposition 2.1.

- Soient des entiers naturels $a, b, c, j, k, \ell, p, q^{\prime}, s^{\prime}$ vérifiant

$1 \leq a+b+c \leq 3 \quad$ et $\quad 1 \leq j \leq k \leq \ell \leq \ell+p \leq \ell+p+q^{\prime} \leq \ell+p+s^{\prime}$.

En désignant par $\lfloor$.$\rfloor la partie entière, posons$

$$
\begin{aligned}
& A:=\left(\sum_{i=1}^{j-1} X_{-\left(\left\lceil\frac{1}{2} p\right\rceil+\ell-i\right)}, X_{-\left(\left\lceil\frac{1}{2} p\right\rceil+\ell-j\right)}, X_{-\left(\left\lceil\frac{1}{2} p\right\rceil+\ell-k\right)}, X_{-\left\lceil\frac{1}{2} p\right\rceil}\right) \\
& B:=X_{\left\lfloor\frac{1}{2} p\right\rfloor}{ }^{a} X_{\left\lfloor\frac{1}{2} p\right\rfloor+q^{\prime}}{ }^{b} X_{\left\lfloor\frac{1}{2} p\right\rfloor+s^{\prime}}{ }^{c} .
\end{aligned}
$$

Soit $F: \mathbb{R}^{4} \rightarrow \mathbb{R}$ une fonction différentiable. La quantité

$$
\operatorname{Cov}_{\nu}\left(F\left(S_{j-1}, X_{j}, X_{k}, X_{\ell}\right), X_{\ell+p}{ }^{a} X_{\ell+p+q^{\prime}}{ }^{b} X_{\ell+p+s^{\prime}}{ }^{c}\right)
$$

est alors égale à $\operatorname{Cov}_{\nu}(F(A), B)$. Comme la mesure $\nu$ est dans $E(0)$, il suffit de montrer que, pour tout $\ell$ dans $E(0)$, nous avons

$$
\begin{aligned}
\mid \ell(F(A) B) & -\ell(F(A)) \nu(B) \mid \\
\leq & \leq C\left(\ell(|F(A)|)+\ell\left(\sup _{|u|_{\infty} \leq M}|D F(A+u)|_{\infty}\right)\right) \xi_{p, s^{\prime}} .
\end{aligned}
$$

Par définition de $E(0)$, il suffit de montrer (12) dans le cas où $\ell$ est dans $E_{1}(0)$. Soit un élément $V$ de $\mathcal{V}$. Montrons que (12) est vraie pour $\ell=\ell_{V}$.

- D'après le premier point de la démonstration, pour tout $\omega, \omega^{\prime} \in V$, nous avons

$$
\left|A(\omega)-A\left(\omega^{\prime}\right)\right|_{\infty} \leq C_{f}^{(\eta)} \sum_{j \geq\left\lceil\frac{1}{2} p\right\rceil}\left(C_{4} \rho_{4}^{j}\right)^{\eta}=C_{f}^{(\eta)} C_{4}^{\eta} \frac{\rho_{4}^{\frac{1}{2} \eta p}}{1-\rho_{4} \eta}
$$

et donc

$$
\begin{aligned}
\mid \ell_{V}([F(A) & \left.\left.-\ell_{V}(F(A))\right] B\right) \mid \\
& \leq\|f\|_{\infty}^{a+b+c} 3 \ell_{V}\left(\sup _{|u|_{\infty} \leq M}|D F(A(.)+u)|_{\infty}\right) C_{f}^{(\eta)} C_{4}{ }^{\eta} \frac{\rho_{4}{ }^{\frac{1}{2} \eta p}}{1-\rho_{4}{ }^{\eta}} .
\end{aligned}
$$

TOME $133-2005-\mathrm{N}^{\mathrm{O}} 3$ 
- Pour conclure, nous utilisons le même argument que celui utilisé par Dolgopyat pour prouver les corollaires 6.2 et 6.3 de [8]. Soit un élément $V^{\prime}$ appartenant à $\mathcal{V}$. Nous notons $\pi_{V}$ et $\pi_{V^{\prime}}$ les projections $: \pi_{V}: V \times[0,1] \rightarrow V$ et $\pi_{V^{\prime}}: V^{\prime} \times[0,1] \rightarrow V^{\prime}$. Nous avons

$$
\begin{aligned}
& \left|\ell_{V}(B)-\ell_{V^{\prime}}(B)\right| \\
& \quad=\left|\int_{V \times[0,1]} B(x) \mathrm{d} m_{V}(x, t)-\int_{V^{\prime} \times[0,1]} B\left(x^{\prime}\right) \mathrm{d} m_{V^{\prime}}\left(x^{\prime}, t^{\prime}\right)\right| \\
& \quad \leq \int_{V \times[0,1]}\left|B\left(\pi_{V}(y)\right)-B\left(\pi_{V^{\prime}}\left(\tau_{V, V^{\prime}}(y)\right)\right)\right| \mathrm{d} m_{V}(y) .
\end{aligned}
$$

Notons $Z(p):=\left\{y \in V \times[0,1] ; R_{V, V^{\prime}}(y)<\frac{1}{2}\left\lfloor\frac{1}{2} p\right\rfloor\right\}$.

D'après la proposition 2.1 , pour tout $y \in Z(p)$ et tout $j \in\left\{0, q^{\prime}, s^{\prime}\right\}$ nous avons

$$
\begin{aligned}
\left|X_{\left\lfloor\frac{1}{2} p\right\rfloor+j}\left(\pi_{V}(y)\right)-X_{\left\lfloor\frac{1}{2} p\right\rfloor+j}\left(\pi_{V^{\prime}}\left(\tau_{V, V^{\prime}}(y)\right)\right)\right| & \leq C_{f}^{(\eta)}\left(C_{1} \rho_{1}^{\frac{1}{2}\left\lfloor\frac{1}{2} p\right\rfloor}\right)^{\eta} \\
& \leq C_{f}^{(\eta)} C_{1}^{\eta} \rho_{1}^{-\frac{1}{2} \eta} \rho_{1}^{\frac{1}{4} p \eta} .
\end{aligned}
$$

Nous avons donc

$$
\begin{aligned}
& \int_{Z(p)}\left|B\left(\pi_{V}(y)\right)-B\left(\pi_{V^{\prime}}\left(\tau_{V, V^{\prime}}(y)\right)\right)\right| \mathrm{d} m_{V}(y) \\
& \leq(a+b+c)\|f\|_{\infty}^{a+b+c-1} C_{f}^{(\eta)} C_{1}^{\eta} \rho_{1}^{-\frac{1}{2} \eta} \rho_{1}^{\frac{1}{4} p \eta}
\end{aligned}
$$

et

$$
\begin{array}{r}
\int_{V \times[0,1] \backslash Z(p)}\left|B\left(\pi_{V}(y)\right)-B\left(\pi_{V^{\prime}}\left(\tau_{V, V^{\prime}}(y)\right)\right)\right| \mathrm{d} m_{V}(y) \\
\leq 2\|f\|_{\infty}^{a+b+c} C_{2} \frac{\rho_{2} \frac{1}{4} p}{\rho_{2}^{\frac{3}{4}}} .
\end{array}
$$

- Comme $\nu$ est dans $E(0)$, nous déduisons de ce qui précède

(15) $\left|\ell_{V}(F(A))\left(\ell_{V}(B)-\nu(B)\right)\right|$

$$
\leq \ell_{V}(F(A))\left\{\left(1+3\|f\|_{\infty}^{2}\right) C_{f}^{(\eta)} C_{1}^{\eta} \rho_{1}^{-\frac{1}{2} \eta} \rho_{1}^{\frac{1}{4} p \eta}+2\left(1+\|f\|_{\infty}^{3}\right) C_{2} \frac{\rho_{2}^{\frac{1}{4} p}}{\rho_{2} 2^{\frac{3}{4}}}\right\} .
$$

Ce qui conclut la démonstration du théorème 4 .

\section{BIBLIOGRAPHIE}

[1] Blank (M.), Keller (G.) \& Liverani (C.) - Ruelle-Perron-Frobenius spectrum for Anosov maps, Nonlinearity, t. 15 (2002), pp. 1905-1973.

[2] Bolthausen (E.) - Exact convergence rates in some martingale central limit theorems, Ann. Probab., t. 10 (1982), pp. 672-688.

[3] Bressaud (X.) \& Liverani (C.) - Anosov diffeomorphisms and coupling, Ergodic Theory Dynam. Systems (à paraître).

BUlletin DE LA SOCiÉtÉ MATHÉmATiQUe DE FRANCE 
[4] Conze (J.-P.) \& Le Borgne (S.) - Méthode de martingales et flot géodésique sur une surface de courbure négative constante, Ergodic Theory Dynam. Systems, t. 21 (2001), pp. 421-441.

[5] — Propriétés statistiques des systèmes dynamiques et méthode des martingales, l'exemple des nilvariétés, en préparation.

[6] Denker (M.) \& PhilipP (W.) - Approximation by Brownian motion for Gibbs measures and flows under a function, Ergodic Theory Dynam. Systems, t. 4 (1984), pp. 541-552.

[7] Dolgopyat (D.) - On decay of correlations in Anosov flows, Ann. of Math. (2), t. 147 (1998), pp. 357-390.

[8] - On dynamics of mostly contracting diffeomorphisms, Comm. Math. Physics, t. 213 (2000), pp. 181-201.

[9] _ Limit theorems for partially hyperbolic systems, preprint.

[10] Esseen (C.) - Fourier analysis of distribution functions. A mathematical study of the Laplace-Gaussian Law, Acta Math., t. 77 (1945), pp. 1-125.

[11] Field (M.), Melbourne (I.) \& TöröK (A.) - Decay of correlations, central limit theorems and approximation by Brownian motion for compact Lie group extensions, Ergodic Theory Dynam. Systems, t. 23 (2003), pp. 87-110.

[12] Guivarc'H (Y.) \& Hardy (J.) - Théorèmes limites pour une classe de chaînes de Markov et applications aux difféomorphismes d'Anosov, Ann. Inst. H. Poincaré Probab. Statist., t. 24 (1988), pp. 73-98.

[13] Haeusler (E.) - On the rate of convergence in the central limit theorem for martingales with discrete and continuous time, Ann. Probab., t. 16 (1988), pp. 275-299.

[14] Hennion (H.) \& Hervé (L.) - Limit theorems for Markov chains and stochastic properties of dynamical systems by quasi-compactness, Lect. Notes in Math., vol. 1766, Springer-Verlag, Berlin, 2001.

[15] JAN (C.) - Vitesse de convergence dans le TCL pour des chaînes de Markov et certains processus associés à des systèmes dynamiques, C. R. Acad. Sci. Paris Sér. I Math., t. 331 (2000), no. 5, pp. 395-398.

[16] _ Rates of convergence for some processes under mixing conditions and application to random matrix products, prépublication, 2001.

[17] _ Vitesse de convergence dans le TCL pour des processus associés à des systèmes dynamiques et aux produits de matrices aléatoires, Thèse, Université de Rennes 1, 2001.

[18] KATOK (A.) \& SpAtzier (R. J.) - First cohomology of Anosov actions of higher rank abelian groups and applications to rigidity, Publ. Math. Inst. Hautes Études Sci., t. 79 (1994), pp. 131-156.

[19] Le Borgne (S.) - Principes d'invariance pour les flots diagonaux sur $\mathrm{SL}(d, \mathbb{R}) / \mathrm{SL}(d, \mathbb{Z})$, Ann. Inst. H. Poincaré Probab. Statist., t. 38 (2002), no. 4 , pp. 581-612. 
[20] Le Borgne (S.) \& PÈne (F.) - Vitesse dans le théoreme limite central pour certains processus stationnaires fortement décorrélés, arXiv : math. PR/0306083, 2003.

[21] LE JAN (Y.) - The central limit theorem for the geodesic flow on noncompact manifolds of constant negative curvature, Duke Math. J., t. 74 (1994), pp. 159-175.

[22] Lind (D.A.) - Dynamical properties of quasihyperbolic toral automorphisms, Ergodic Theory Dynamical Systems, t. 2 (1982), pp. 49-68.

[23] Melbourne (I.) \& TöröK (A.) - Central limit theorems and invariance principles for time-one maps of hyperbolic flows, Comm. Math. Phys., t. 229 (2002), pp. 57-71.

[24] PÈne (F.) - Applications des propriétés stochastiques des systèmes dynamiques de type hyperbolique : ergodicité du billard dispersif dans le plan, moyennisation d'équations différentielles, perturbées par une flot ergodique, Thèse, Université de Rennes, 2000.

[25] _ Averaging method for differential equations perturbed by dynamical systems, prépublication 01-37, IRMAR, Université de Rennes, 2001.

[26] - Rates of convergence in the CLT for two-dimensional dispersive billiards, Comm. Math. Phys., t. 225 (2002), no. 1, pp. 91-119.

[27] RATnER (M.) - The central limit theorem for geodesic flows on $n$ dimensional manifolds of negative curvature, Israel J. Math., t. 16 (1973), pp. 181-197.

[28] Rio (E.) - Sur le théorème de Berry-Esseen pour les suites faiblement dépendantes, Probab. Th. Relat. Fields, t. 104 (1996), pp. 255-282.

[29] Shub (M.) \& Wilkinson (A.) - Pathological foliations and removable zero exponents, Invent. Math., t. 139 (2000), pp. 495-508.

[30] Sinaï (JA.G.) - The central limit theorem for geodesic flows on manifolds of constant negative curvature, Dokl. Akad. Nauk SSSR, t. 133 (1960), pp. 1303-1306, en russe; trad. anglaise dans Soviet Math. Dokl. t. 1 (1960), pp. $983-987$.

[31] Young (L.-S.) - Statistical properties of dynamical systems with some hyperbolicity, Ann. of Math. (2), t. 147 (1998), pp. 585-650. 